\title{
Análisis del estado poblacional del yodo en Colombia y la necesidad de modificar los niveles de yodación universal de la sal
}

\author{
Analysis of the population status of iodine in Colombia and the need to \\ modify the levels of universal iodization of salt
}

Vargas Uricoechea $H,{ }^{1}$ Murillo Palacios $J,{ }^{2}$ Ramírez Bejarano $L E^{2}$

\begin{abstract}
${ }^{1} \mathrm{MD}$; MSc; PhD. Director del grupo de estudio de enfermedades metabólicas y profesor asociado del Departamento de Medicina Interna, Facultad de Ciencias de la Salud, Universidad del Cauca. Popayán, Colombia.

${ }^{2} \mathrm{MD}$; Especialista en medicina interna; grupo de estudio de enfermedades metabólicas, Universidad del Cauca. Popayán, Colombia.
\end{abstract}

Autor de correspondencia: Hernando Vargas Uricoechea Correo electrónico: hernandovargasuricoechea@gmail.com Fecha de recepción: 15/10/2019

Fecha de aceptación: 16/12/2019

\section{Resumen}

La deficiencia y el exceso de yodo tienen graves consecuencias para la salud humana y se han asociado con la presencia de bocio, hipotiroidismo, hipertiroidismo, cáncer de tiroides, nódulos y autoinmunidad tiroideos, así como un pobre desarrollo mental e intelectual. Los programas de yodación universal de la sal se han considerado como una de las intervenciones más rentables para la prevención de los trastornos asociados con la deficiencia de yodo; sin embargo, estos esfuerzos también han llevado a un uso excesivo de yodo en ciertas áreas geográficas, de acuerdo con el uso excesivo de sal. Como tal, la cantidad de yodo derivada del uso de la sal excede los límites que se establecen para lograr un equilibrio entre su consumo y los beneficios para la salud, lo cual conduce a efectos indeseables para la salud. En Colombia, se cumplen totalmente las recomendaciones y normas para la producción y comercialización de la sal yodada. Sin embargo, existe un notable exceso de yodo entre la población, lo cual, al menos hipotéticamente, representa un mayor riesgo para el desarrollo de trastornos funcionales y estructurales de la glándula tiroides. Esta revi- sión analiza los programas universales de yodación de la sal, particularmente, su impacto en la tiroides, y se centra en datos de salud pública de Colombia, en respuesta a la implementación de dicha estrategia.

Palabras clave: yodo, tiroides, bocio, autoinmunidad, yodo en orina.

\section{Abstract}

Iodine deficiency and iodine excess have severe consequences on human health, and have been associated with the presence of goiter, hypothyroidism, hyperthyroidism, thyroid cancer, thyroid nodules and thyroid autoimmunity, as well as poor mental and intellectual development. Universal salt iodization programs have been considered one of the most cost-effective interventions for the prevention of iodine deficiency-associated disorders, as evidenced over time since the implementation of such programs. However, these efforts have also led to an excessive use of iodine in certain geographical regions, according to the overuse of salt. As such, the amount of iodine derived from the use of salt exceeds the established limits that are set to strike a balance between salt consumption and health benefits, thereby leading to undesirable health effects. In Colombia, the recommendations and standards for the production and commercialization of iodized salt are fully complied with. Nevertheless, there is a remarkable rate of iodine excess among the country's population, which, at least hypothetically, represents a higher risk for developing functional and structural disorders of the thyroid gland. This review analyzes universal salt iodization programs, particularly, their impact on the thyroid and with focus on public health data from Colombia in response to the implementation of this strategy.

Keywords: iodine, thyroid, goiter, autoimmunity, urinary iodine. 


\section{Introducción}

El impacto clínico asociado con la deficiencia o el exceso en la ingesta de yodo es uno de los mayores problemas en salud pública del mundo; los desórdenes por deficiencia de yodo (DDY) han sido los más estudiados y los que mayor esfuerzo han suscitado por parte de los diferentes organismos regulatorios internacionales $(1,2)$. La deficiencia de yodo es un fenómeno que ha acompañado a la humanidad a lo largo de la historia, al igual que sus consecuencias como hipotiroidismo, incremento en la mortalidad perinatal, retardo mental, bocio, entre otras. A pesar de los esfuerzos realizados en las últimas décadas con el objetivo de eliminar los DDY, aún persiste un número aproximado de 2 mil millones de personas distribuidas en todo el mundo con alto riesgo de padecerlos. En aquellas áreas geográficas con deficiencia de yodo, los programas de yodación universal de la sal (YUS) son la mejor estrategia desde el punto de vista costo-efectivo para el control de dichos desórdenes $(3,4)$.

La sal es un ingrediente que se consume de manera universal y en cantidades relativamente constantes a lo largo del año, lo cual explica las razones por las que se justifica utilizarla como vehículo para el suministro de yodo. Adicionalmente, el proceso de yodación de la sal es sencillo y de bajo costo; por ello, un número considerable de países han determinado el carácter obligatorio de la YUS como estrategia para la eliminación de los DDY. No obstante; los indicadores utilizados para monitorizarlos no se llevan a cabo de forma sistemática y actualmente se ha encontrado que la distribución mundial de los trastornos asociados con la ingesta de yodo ha cambiado, con un aumento importante de áreas geográficas con alta prevalencia de ingesta de yodo catalogada como más que adecuada o excesiva $(5,6)$.
Asimismo, el exceso en la ingesta se ha relacionado con autoinmunidad tiroidea (hipotiroidismo e hipertiroidismo autoinmune), bocio, enfermedad nodular tiroidea, entre otros (Tabla 1). En 2017, Colombia se estableció como uno de los 11 países identificados con ingesta excesiva de yodo. Este cambio en la distribución de los trastornos asociados con la ingesta de yodo (en aquellas áreas con antecedentes de deficiencia y que actualmente se comportan como áreas con exceso de yodo) permite predecir que la frecuencia de otras patologías tiroideas asociadas puede incrementarse $(7,8)$.

A pesar de lo anterior, se considera que el beneficio sobre la salud que proveen los programas de YUS sobrepasa el riesgo de disfunción tiroidea que puede originar dicha intervención. Los criterios para la evaluación de la nutrición de yodo en la población se muestran en la Tabla 2.

\section{Ingesta de yodo y sus efectos en la tiroides}

El mecanismo por el cual el exceso de yodo origina disfunción tiroidea no está bien establecido; sin embargo, induce la producción de citocinas y quimiocinas, las cuales pueden reclutar células inmunocompetentes a la tiroides, y junto al procesamiento del exceso de yodo a nivel intratiroideo origina un incremento del estrés oxidativo, aumentando la oxidación lipídica y el daño tisular; también la incorporación del yodo a la tiroglobulina (Tg) la vuelve más antigénica, lo que genera un mayor riesgo de autoinmunidad tiroidea $(9,10)$.

La tiroides tiene una adaptación intrínseca al exceso de yodo llamada efecto agudo de Wolff-Chaikoff, que puede explicarse por la generación de sustancias inhibidoras (yodolactonas, yodoaldehídos y yodolípidos), que actúan sobre la actividad de la peroxidasa tiroidea (TPO), con una posterior disminución en la actividad de las desyodinasas intratiroideas,

Tabla 1. Consecuencias sobre la salud poblacional de la deficiencia o del exceso de yodo

\begin{tabular}{l|l}
\multicolumn{1}{c|}{ Déficit en la ingesta de yodo } & \multicolumn{1}{c}{ Exceso en la ingesta de yodo } \\
\hline Hipotiroidismo & Hipertiroidismo \\
\hline Bocio, hipotiroidismo y enfermedad nodular tiroidea & Incremento en el riesgo de autoinmunidad tiroidea \\
\hline $\begin{array}{l}\text { Incremento en el riesgo de cáncer de tiroides } \\
\text { Incremento en la de susceptibilidad de la tiroides a la radia- } \\
\text { ción nuclear }\end{array}$ & Hipertiroidismo inducido por yodo (efecto de Jod-Basedow) \\
\hline $\begin{array}{l}\text { Incremento en la tasa de abortos, mortalidad fetal, perinatal } \\
\text { e infantil }\end{array}$ & Bocio \\
\hline $\begin{array}{l}\text { Cretinismo endémico por yodo (efecto de Wolff-Chaikoff) } \\
\text { de la capacidad intelectual y laboral }\end{array}$ & Probable incremento en la mortalidad de origen cardiovascular \\
\hline
\end{tabular}


Tabla 2. Criterios de evaluación nutricional de yodo poblacional, según la mediana o rango de la yoduria

\begin{tabular}{|c|c|c|}
\hline & $\begin{array}{l}\text { Ingesta de } \\
\text { yodo }\end{array}$ & $\begin{array}{l}\text { Aporte nutricional de } \\
\text { yodo }\end{array}$ \\
\hline \multicolumn{3}{|c|}{ Niños en edad escolar } \\
\hline$<20 \mu \mathrm{g} / \mathrm{L}$ & Insuficiente & Deficiencia grave de yodo \\
\hline $20-49 \mu \mathrm{g} / \mathrm{L}$ & Insuficiente & $\begin{array}{l}\text { Deficiencia moderada de } \\
\text { yodo }\end{array}$ \\
\hline $50-99 \mu \mathrm{g} / \mathrm{L}$ & Insuficiente & Deficiencia leve de yodo \\
\hline $100-299 \mu \mathrm{g} / \mathrm{L}$ & Adecuada & $\begin{array}{l}\text { Nutrición adecuada de } \\
\text { yodo }\end{array}$ \\
\hline$\geq 300 \mu \mathrm{g} / \mathrm{L}$ & Excesiva & $\begin{array}{l}\text { Ingesta excesiva de yodo } \\
\text { (riesgo de consecuen- } \\
\text { cias perjudiciales para la } \\
\text { salud [hipertiroidismo, } \\
\text { enfermedad autoinmune } \\
\text { tiroidea]) }\end{array}$ \\
\hline \multicolumn{3}{|c|}{ Mujeres embarazadas } \\
\hline$<150 \mu \mathrm{g} / \mathrm{L}$ & Insuficiente & - \\
\hline $150-249 \mu \mathrm{g} / \mathrm{L}$ & Adecuada & - \\
\hline $250-499 \mu \mathrm{g} / \mathrm{L}$ & $\begin{array}{l}\text { Más que } \\
\text { adecuada } \\
\text { (superior a las } \\
\text { necesidades) }\end{array}$ & - \\
\hline$\geq 500 \mu \mathrm{g} / \mathrm{L}$ & Excesiva* & - \\
\hline \multicolumn{3}{|c|}{ Mujeres lactando** } \\
\hline$<100 \mu \mathrm{g} / \mathrm{L}$ & Insuficiente & - \\
\hline$\geq 100 \mu \mathrm{g} / \mathrm{L}$ & Adecuada & - \\
\hline \multicolumn{3}{|l|}{ Niños $<2$ años } \\
\hline$<100 \mu \mathrm{g} / \mathrm{L}$ & Insuficiente & - \\
\hline$\geq 100 \mu \mathrm{g} / \mathrm{L}$ & Adecuada & - \\
\hline
\end{tabular}

*El término excesiva significa que excede a la cantidad requerida para prevenir y controlar el déficit de yodo. ${ }^{* *}$ En mujeres lactando, los números para la mediana de la yoduria son más bajos que los requerimientos de yodo, debido a la excreción de yodo por la leche materna.

lo que reduce la síntesis de hormonas tiroideas $(11,12)$. En la mayoría de los individuos sometidos a un exceso de yodo de rápida instauración, este efecto es transitorio y se recupera después de generarse otro fenómeno denominado escape $o$ adaptación al efecto agudo de Wolff-Chaikoff, el cual se asocia con la disminución en la expresión del importador sodio/yodo (SNI), el cual es el mediador del trasporte activo de yodo desde la circulación hacia la célula tiroidea. La disminución en la expresión del SNI ocurre alrededor de las 24 horas posteriores al exceso agudo de yodo, causando la disminución en la concentración del yodo intratiroideo y en los niveles de sustancias yodinadas; de este modo, se inhibe la síntesis de hormonas tiroideas e induce posteriormente el reinicio de su producción en condiciones normales $(13,14)$.

En individuos vulnerables, como aquellos con anticuerpos tiroideos, antecedente de tiroiditis, uso de yodo radioactivo o medicamentos como amiodarona, interferón $\alpha$, litio, puede fallar el fenómeno de escape al efecto agudo de Wolff-Chaikoff y tienen un incremento en el riesgo de hipotiroidismo permanente. De igual manera, en pacientes susceptibles como los que tienen bocio nodular no tóxico, enfermedad de Graves-Basedow (EGB) latente o en áreas con deficiencias prolongadas y graves de yodo), el exceso de este elemento puede inducir hipertiroidismo (fenómeno de Jod-Basedow). Este desorden metabólico es relativamente frecuente en áreas donde la ingesta de yodo es muy alta; de hecho, en aquellos sitios donde el consumo es marginal (pero la deficiencia está ausente), los incrementos moderados en la ingesta pueden inducir hipertiroidismo en individuos con enfermedad nodular tiroidea autónoma (15-17).

Por su parte, se ha demostrado un incremento en la frecuencia de cáncer bien diferenciado de tiroides (CBDT) en sitios donde se han implementado programas de YUS; pero a pesar de esto también se ha evidenciado un incremento en la frecuencia de este tipo de cáncer en otros países con baja ingesta. En general, la deficiencia de yodo se relaciona con un incremento del riesgo de CBDT (especialmente, la variedad folicular) y probablemente de cáncer anaplásico, mientras que una alta ingesta de yodo (especialmente, en áreas deficientes) se relaciona con una reducción no significativa del riesgo de cáncer tiroideo; adicionalmente, también se ha encontrado una probable asociación entre la presencia de autoanticuerpos tiroideos como anti-Tg y la elevación en los niveles de TSH con CBDT (específicamente, el tipo papilar). En modelos animales, se ha documentado que tanto el exceso como el déficit de yodo incrementan la proliferación de células tiroideas y la formación de adenomas tiroideos, pero no el riesgo de carcinogénesis; sin embargo, cuando se exponen a radiación se ha demostrado que ambas situaciones incrementan el riesgo de cáncer tiroideo, lo que plantea la hipótesis de que el exceso y la deficiencia pueden considerarse promotores más que inductores de neoplasia. Asimismo, la deficiencia de yodo también promueve la generación de especies reactivas de oxígeno, que producen daño directo del ácido desoxirribonucleico (ADN), apoptosis y mayor riesgo de mutaciones (18-20).

\section{Historia y marco conceptual del estado poblacional del yodo en Colombia}

Solo hasta 1948 se conoció la prevalencia del bocio endémico en Colombia, la cual fue de 53 \% (a partir de una mues- 
tra nacional en 183243 escolares), la prevalencia fue mayor en departamentos como Caldas y Cauca (>80 \%). En 1947 se estableció la yodación de la sal para el consumo humano y en 1950 se inició un proyecto de yodación de la sal, con una gran producción destinada a los municipios que habían presentado las prevalencias más altas de bocio endémico para la época. El Instituto Nacional de Nutrición (INN) evaluó tal intervención en 1952, en un estudio realizado en 6511 escolares, y encontró una prevalencia de bocio del 33 \%. En 1955 se reglamentó que el contenido de yodo en la sal podía fluctuar entre 50-100 partes por millón (ppm), que es lo mismo que decir 50-100 mg de yodo/kilogramo de sal (21-23). En 1960, el INN junto con el Comité Interdepartamental de Nutrición para la Defensa Nacional de Estados Unidos evaluaron a 4818 adultos y 1263 niños, y se encontró una prevalencia de bocio endémico de 39,5\% en hombres y de 43,7 \% en mujeres. En 1965 el INN realizó una encuesta en 12266 escolares que residían en 8 municipios del departamento de Caldas (donde la prevalencia había sido $>80 \%$ en 1948) y se documentó una prevalencia de bocio $<2 \%$. La eficacia de la intervención poblacional a través de la YUS se comprobó en el estudio nacional de salud (1977-1980) donde se encontró una prevalencia de bocio endémico $<2 \%$.

A comienzos de la década de 1980 se comercializaba una sal para consumo humano que no cumplía con las normas establecidas por el Ministerio de Salud, lo que causó que la prevalencia de bocio endémico en sitios como Chámeza y Yopal, en el departamento del Casanare, fuera de $52 \%$ y 13,5\%, respectivamente. En agosto de 1992, el Ministerio de Salud creó un comité para la vigilancia de los DDY y se constituyó un grupo de trabajo conformado por diferentes entes académicos con el propósito de establecer un protocolo de investigación para evaluar la prevalencia de los DDY, y que fue aprobado en 1993. Con la apertura económica, la sal se convirtió en un producto de libre comercialización, lo que permitió la introducción de sal no yodada (o la mezcla de sal yodada con no yodada); en consecuencia, en 1993 la prevalencia de bocio se incrementó al $15 \%$ en algunas regiones del país.

En 1994 se reglamentaron las funciones del Instituto Nacional de Vigilancia de Medicamentos y Alimentos (INVIMA), que asumió el desarrollo del programa nacional de control de calidad de la sal. En 1996 se reglamentó la expedición del registro sanitario y las condiciones sanitarias de producción, empaque, comercialización y control de la sal (24-26). Adicionalmente, la sal fue clasificada como alimento y se ratificó que debía contener yodo (como yoduro) en proporción de 50100 ppm. En el mismo año se implementó el Plan Nacional de Alimentación y Nutrición 1996-2005, en el que se definió la prevención y control de las deficiencias de micronutrientes, y se propuso mejorar los programas de vigilancia y control de la sal yodada; en reconocimiento de lo anterior, el 29 de abril de 1998 se llevó a cabo el acto de declaratoria de Colombia como país "Libre de DDY" por parte de las agencias internacionales de salud, el Fondo de las Naciones Unidas para la Infancia (UNICEF), la Organización Mundial de la Salud/Organización Panamericana de la Salud (OMS/OPS) y el Consejo Internacional para el Control de los DDY (ICCIDD) $(27,28)$.

\section{Situación de los programas de YUS en América Latina}

Una cantidad importante de países han establecido por ley la YUS, por ejemplo, Costa Rica, Guatemala, México, República Dominicana y Venezuela, que utilizan yodato de potasio $\left(\mathrm{KIO}_{3}\right)$ y yoduro de potasio (KI). Sin embargo, sigue existiendo un rango muy amplio con respecto a los niveles de yodación, que oscila entre 15-100 ppm. Hasta el momento, en 9 países se establece que más del $70 \%$ de la sal yodada se procesa con tecnología de punta y con un alto estándar de calidad, mientras otros 9 países realizan el proceso a partir de tecnologías que no garantizan un control de calidad adecuado $(29,30)$. Finalmente, en 11 países, más del $90 \%$ de los hogares consume sal adecuadamente yodada ( $\geq 15 \mathrm{ppm}$ ), mientras que en 4 países la cobertura es del 80-89\%; por su parte, en países como El Salvador, Guatemala y Venezuela, la cobertura se encuentra entre el 54-79 \% (Tabla 3).

Aunque todavía en algunos países hay ausencia de programas de monitorización de la calidad de la yodación de la sal, el porcentaje de hogares en los que más del $80 \%$ consume adecuadamente sal yodada se incrementó del $76 \%$ al $83 \%$, y la cobertura de hogares con sal adecuadamente yodada en toda América Latina aumentó de 84,7 \% al 90,6\%, desde el período de 1995-2004 al período de 2005-2013.

\section{Estudios que han evaluado los DDY en Colombia antes de la declaratoria como país libre de DDY}

Entre los años 1948 y 1993 se realizaron 7 estudios (3 en escolares y 4 en población general) en áreas tanto urbanas como rurales. Hasta entonces se establecía únicamente la prevalencia de bocio por medio de la palpación como estrategia para determinar el estado poblacional del yodo y no se medía la yoduria como parámetro principal para la evaluación de los trastornos asociados con la ingesta de yodo. La prevalencia de bocio disminuyó radicalmente desde 1948 hasta 1977-1980; posteriormente, hubo un incremento a comienzos de la década de 1980 atribuido a la comercialización de sal para consumo humano que no cumplía las normas del Ministerio de Salud (31-33). De esta forma, la prevalencia de bocio endémico tanto en áreas urbanas como rurales se incrementó significativamente, en compañía de la introducción de un mercado paralelo de sal inadecuadamente yodada, que abarcó el $50 \%$ del total nacional; esto indica que los programas de 
prevención desarrollados inadecuadamente incrementan el riesgo de DDY (Tabla 4).

\section{Estudios que han evaluado los DDY en Colombia después de la declaratoria como país libre de DDY}

En el estudio de prevalencia de los DDY e ingestión promedio de sal, Colombia 1994-1998, se evaluó la prevalencia de bocio, yoduria y contenido de yodo en la sal, y se encontró una prevalencia de bocio grado I del 6,5 \%; las mayores prevalencias se presentaron en regiones como Santander y Norte de Santander (20,6 \%), Tolima, Huila, Caquetá (16,7 \%) y Bogotá (11,2\%). Más del $90 \%$ de las yodurias eran >100 $\mu \mathrm{g} / \mathrm{L}$ y el consumo promedio persona-día de sal fue de 11,42 g. Posteriormente, se llevaron a cabo varios estudios centinelas en los que se encontró que gran parte de la población tenía una ingesta excesiva de yodo, como los estudios en escolares

Tabla 3. Aspectos básicos sobre la legislación, nivel de yodación, compuestos utilizados para la yodación de la sal y consumo de sal yodada en América Latina

\begin{tabular}{|c|c|c|c|c|c|c|c|c|c|c|}
\hline \multirow[t]{3}{*}{ País } & \multicolumn{2}{|c|}{ Legislación } & \multirow{3}{*}{$\begin{array}{l}\text { Nivel de } \\
\text { yodación } \\
\text { (ppm) }\end{array}$} & \multirow{3}{*}{$\begin{array}{c}\text { Compuesto } \\
\text { usado para } \\
\text { yodación }\end{array}$} & \multicolumn{2}{|c|}{ Plantas de producción } & \multicolumn{4}{|c|}{ Consumo de sal yodada en hogares } \\
\hline & \multirow[t]{2}{*}{ Humana } & \multirow[t]{2}{*}{ Animal } & & & \multirow{2}{*}{$\begin{array}{c}\text { Gran- } \\
\text { des (\%) }\end{array}$} & \multirow{2}{*}{$\begin{array}{c}\text { Pequeñas/ } \\
\text { medianas } \\
(\%)\end{array}$} & \multirow{2}{*}{$\begin{array}{l}\text { Encuesta de } \\
\text { frecuencia }\end{array}$} & \multicolumn{3}{|c|}{ Último control } \\
\hline & & & & & & & & Año & $\begin{array}{l}\text { Exten- } \\
\text { sión }\end{array}$ & $\begin{array}{c}\geq 15 \text { ppm } \\
(\%)\end{array}$ \\
\hline Argentina & Sí & $\mathrm{Si}^{*}$ & $25-40$ & $\mathrm{KIO}_{3}$ & 93 & 7 & Esporádica & $\begin{array}{l}2009- \\
2012\end{array}$ & $\mathrm{R}$ & 86 \\
\hline Belice & Sí & & & & & & Esporádica & & & \\
\hline Bolivia & Sí & & $40-80$ & $\mathrm{KIO}_{3}$ & 38 & 62 & Esporádica & 2008 & $\mathrm{~N}$ & 89 \\
\hline Brasil & Sí & & $15-45$ & $\mathrm{KIO}_{3}$ & & & Esporádica & 2006 & $\mathrm{~N}$ & 96 \\
\hline Chile & Sí & & $20-60$ & $\mathrm{KIO}_{3}$ & 90 & 10 & Esporádica & 2011 & $\mathrm{~N}$ & 95 \\
\hline Colombia & Sí & & $50-100$ & $\mathrm{KIO}_{3}$ & & & Esporádica & 2005 & $\mathrm{~N}$ & 97 \\
\hline Costa Rica & Sí & & $30-60$ & $\mathrm{KIO}_{3} / \mathrm{KI}$ & & 100 & Esporádica & 2008 & $\mathrm{~N}$ & 97 \\
\hline Cuba & Sí & $\mathrm{Si}^{*}$ & $20-30$ & & 100 & & Semanal & 2012 & $\mathrm{~N}$ & 88 \\
\hline $\begin{array}{l}\text { República } \\
\text { Dominicana }\end{array}$ & Sí & $\mathrm{Si}^{*}$ & $30-100$ & $\mathrm{KIO}_{3} / \mathrm{KI}$ & & & & & & \\
\hline Ecuador & Sí & $\mathrm{Si}^{*}$ & $20-40$ & $\mathrm{KIO}_{3}$ & 75 & 25 & Anual & 2013 & $\mathrm{~N}$ & 93 \\
\hline El Salvador & Sí & $\mathrm{Si}^{*}$ & $30-100$ & $\mathrm{KIO}_{3}$ & & 100 & Bienal & 2012 & $\mathrm{~N}$ & 74 \\
\hline Guatemala & Sí & $\mathrm{Si}^{*}$ & $20-60$ & $\mathrm{KIO}_{3} / \mathrm{KI}$ & 60 & 40 & Esporádica & 2010 & $\mathrm{~N}$ & 54 \\
\hline \multicolumn{11}{|l|}{ Guyana } \\
\hline Haití & Sí & & $30-50$ & $\mathrm{KIO}_{3}$ & & & & & & \\
\hline Honduras & Sí & & $50-100$ & $\mathrm{KIO}_{3}$ & & & Esporádica & 2009 & $\mathrm{~N}$ & 96 \\
\hline México & Sí & $\mathrm{Si}^{*}$ & $20-40$ & $\mathrm{KIO}_{3} / \mathrm{KI}$ & 95 & 5 & Mensual & 2013 & $\mathrm{~N}$ & $94^{* *}$ \\
\hline Nicaragua & Sí & & $30-60$ & $\mathrm{KIO}_{3}$ & & & Esporádica & 2009 & $\mathrm{~N}$ & 81 \\
\hline Panamá & Sí & Sí & $20-60$ & $\mathrm{KIO}_{3}$ & 80 & 20 & Bienal & 2012 & $\mathrm{~N}$ & 97 \\
\hline Paraguay & Sí & $\mathrm{Si}^{*}$ & $30-50$ & $\mathrm{KIO}_{3}$ & 12 & 88 & Anual & 2013 & $\mathrm{~N}$ & 97 \\
\hline Perú & Sí & $\mathrm{Si}^{*}$ & $30-40$ & $\mathrm{KIO}_{3}$ & 80 & 20 & Anual & 2013 & $\mathrm{~N}$ & 93 \\
\hline Uruguay & Sí & $\mathrm{Si}^{*}$ & $20-40$ & $\mathrm{KIO}_{3}$ & 100 & & Esporádica & 2000 & $\mathrm{~N}$ & $100^{* *}$ \\
\hline Venezuela & Sí & $\mathrm{Si}^{*}$ & $40-70$ & $\mathrm{KIO}_{3} / \mathrm{KI}$ & 80 & 20 & Anual & 2007 & $\mathrm{R}$ & 54 \\
\hline
\end{tabular}

*No se obliga a su cumplimiento. ${ }^{* *}$ Encuestas a nivel de mercado. $\mathrm{KI}$ : yoduro de potasio; $\mathrm{KIO}_{3}$ : yodato de potasio; N: nacional; R: regional; ppm: partes por millón. 
Tabla 4. Estudios que evaluaron los DDY en Colombia antes de la declaratoria como país libre de DDY

\begin{tabular}{|c|c|c|c|c|c|c|c|}
\hline \multirow[t]{2}{*}{ Parámetros evaluados } & \multicolumn{7}{|c|}{ Año } \\
\hline & 1948 & 1952 & 1960 & 1965 & 1977-1980 & 1984-1986 & 1993 \\
\hline Área geográfica & Uy R & Uy R & Uy R & Predominio $\mathrm{R}$ & Uy R & Uy R & Uy R \\
\hline Población & Escolares & Escolares & General & Escolares & General & General & General \\
\hline Yoduria (mediana) & NR & NR & NR & NR & NR & NR & NR \\
\hline $\begin{array}{l}\text { Bocio (prevalencia por } \\
\text { palpación) }\end{array}$ & $53 \%$ & $33 \%$ & $\begin{array}{l}H: 39,5 \% \\
M: 43,7 \%\end{array}$ & $<2 \%$ & $<2 \%$ & $\begin{array}{l}\text { U: } 13,5 \% \\
\text { R: } 52 \%\end{array}$ & $15 \%$ \\
\hline
\end{tabular}

H: hombres; M: mujeres; NR: no reportada; R: rural; U: urbana.

con yodurias en este rango, especialmente en áreas urbanas; aunque también se ha documentado una alta deficiencia de yodo en algunas áreas rurales. De igual forma, la prevalencia de anticuerpos antitiroideos también se encuentra incrementada (31, 34-39).

En los diferentes estudios, se ha encontrado que, en general, la distribución de las medianas de las yodurias tanto en población escolar como en adultos y en embarazadas se ha incrementado desde la implementación de los programas de YUS, con un aumento concomitante en los títulos de anticuerpos antitiroideos y en la prevalencia de bocio. Dichos resultados fueron confirmados a partir de la Encuesta Nacional de la Situación Nutricional en Colombia (ENSIN, 2015), en la que se encontraron los dos extremos de presentación de ingesta de yodo (exceso y deficiencia), siendo preponderantemente mayor y significativa la ingesta excesiva $(70,5 \%$ en mujeres en edad fértil, $75 \%$ en niños de 5-12 años y 63,8 \% en niños de 1-4 años). En el análisis de dicha encuesta, también se concluyó que, en los grupos poblacionales evaluados, el exceso de yodo se podía inferir a partir del exceso de yodo proveniente de la sal (Tablas 5 y 6 ).

\section{Monitorización y evaluación de los programas de YUS y control de los DDY}

En la evaluación de los programas de YUS para el control de los DDY se han establecido algunas preguntas e indicadores basados en algunas preguntas, que se describen a continuación:

Tabla 5. Estudios que han evaluado los niveles de yoduria, prevalencia de bocio y anticuerpos antitiroideos en Colombia, desde la declaratoria como país libre de DDY.

\begin{tabular}{|c|c|c|c|c|c|c|c|c|}
\hline \multirow{2}{*}{$\begin{array}{l}\text { Parámetros } \\
\text { evaluados }\end{array}$} & \multicolumn{8}{|c|}{ Año } \\
\hline & 1994-1998 & 1999 & 2001-2002 & 2006 & 2010 & 2011 & 2013-2014 & 2015 \\
\hline Área geográfica & U & Uy $R$ & Uy $R$ & Uy $R$ & Uy $R$ & U & U & Predominio U \\
\hline Población & Escolares & Escolares & Escolares & Escolares & Escolares & Adultos & Embarazadas & Escolares \\
\hline $\begin{array}{l}\text { Mediana de Yoduria } \\
(\mu \mathrm{g} / \mathrm{L})\end{array}$ & $\begin{array}{l}\geq 100 \text { (en } \\
>90 \% \text { de la } \\
\text { población) }\end{array}$ & $\begin{array}{l}380(U: 705 \\
\text { y R:148) }\end{array}$ & $\begin{array}{l}415(U: 430 \\
\text { y R: } 380)\end{array}$ & $\begin{array}{l}272,4(\mathrm{U}: \\
285,5 \text { y R: } \\
72,6)\end{array}$ & 401,2 & 424,2 & 354 & 510,3 \\
\hline $\begin{array}{l}\text { Bocio (prevalencia } \\
\text { por palpación) }\end{array}$ & $6,5 \%$ & NR & NR & NR & $88 \%$ & NR & $25,5 \%$ & $37,9 \%$ \\
\hline $\begin{array}{l}\text { Anticuerpos antitiroi- } \\
\text { deos (prevalencia) }\end{array}$ & NR & NR & NR & NR & $\begin{array}{l}\text { anti-TPO: } \\
4 \%\end{array}$ & $\begin{array}{l}\text { Anti- } \\
\text { TPO: } \\
\text { 28,9\% }\end{array}$ & $\begin{array}{l}\text { anti-TPO: } \\
0,76 \%\end{array}$ & $\begin{array}{l}\text { anti-TPO: } 42,75 \% \text {; } \\
\text { anti-Tg: } 2,87 \% \text {; } \\
\text { anti-TPO y anti-Tg: } \\
\text { 3,62 \%; TRAb: } 0 \%\end{array}$ \\
\hline
\end{tabular}

Anti-Tg: anticuerpos antitiroglobulina; anti-TPO: anticuerpos antiperoxidasa tiroidea; NR: no reportada; R: rural; TRAb: anticuerpos contra el receptor de tirotropina; U: urbana. 
- ¿Toda la sal que se produce o se importa cumple con los requerimientos de yodación para el país?

- ¿En aquellos países en riesgo de presentar deficiencia de yodo se tiene acceso a la sal adecuadamente yodada y se utiliza en dicha población?

- ¿Existe un grupo en la población que no tiene acceso a la sal yodada y, por tanto, requiere atención?

- ¿Cuál es el impacto de la yodación de la sal y de otras intervenciones sobre el estado de yodo de la población?

- ¿Cuál es la contribución relativa de la ingesta de yodo a partir de diversas fuentes?

Asimismo, en aquellos países donde los programas de erradicación de los DDY no han sido implementados aún, las preguntas a realizar son las siguientes:

- ¿Los DDY han sido eliminados como un problema de salud pública?

- ¿Cuál es la prevalencia de los DDY en grupos poblacionales específicos [embarazadas y niños]?

- ¿Cuáles son los pasos por seguir para dirigir los DDY?

En la misma vía, se han descrito 3 indicadores (de proceso, de impacto y de sostenibilidad) que reflejan la monitorización del contenido de yodo en la sal, el efecto de la yodación de la sal en la población y la eliminación exitosa de los DDY. De igual forma, entre los temas a considerar en los programas que evalúan los DDY se destacan, por ejemplo, el compromiso de los entes políticos, el manejo adecuado de programas de educación y comunicación, la consolidación de sistemas de monitorización y el establecimiento de los objetivos de la suplementación. La integración de cada uno de estos elementos permite que la creación de los programas encaminados a la erradicación de los DDY sea más eficaz y eficiente (Figura 1).

De acuerdo con lo anterior, y desde un punto de vista poblacional, se han descrito los denominados indicadores programáticos para la eliminación sostenida de los DDY (27, 40-42). Se establece que si se logra el $80 \%$ de ellos (8/10), entonces se estarían cumpliendo los objetivos de erradicación de estos trastornos.

\section{Situación actual del estado poblacional del yodo en Colombia y estrategias de prevención de trastornos asociados con la ingesta de yodo}

La principal estrategia para la eliminación sostenida de los DDY sigue siendo la YUS. Las recomendaciones internacionales han sugerido el uso complementario de suplementos de yodo, por ejemplo, aceite yodado, como una medida temporal cuando la yodación no puede ser implementada rápidamente (43-46). Según el reporte de la base global de datos sobre deficiencia de yodo (OMS, 2004), Brasil, Chile, Ecuador, Liberia y Uganda tenían una ingesta excesiva de yodo; sin embargo, los países que en 2017 según la red global de yodo fueron catalogados con ingesta excesiva, en 2004 tenían ingestas muy por debajo de las reportadas posteriormente (31, 47-50). Según este mismo reporte, países como Armenia tenían una ingesta adecuada, mientras que Benín, Colombia, Costa Rica, Honduras y Catar reportaron una ingesta más que adecuada; actualmente, Uganda persiste como el único país que permanece con ingesta excesiva (Tablas 7 y 8 ).

Colombia produce anualmente cerca de 540000 toneladas de sal, lo que garantiza cierta autosuficiencia, por lo que, al considerar la posibilidad de variación de los rangos de yodo en la sal, debe partirse de la premisa de que la ingesta per cápita actual de sal en el país se encuentre en un promedio de 10 g/persona/día. No obstante, es importante considerar que la norma establecida para Colombia de concentración de yodo en la sal (50-100 ppm) es un valor válido a nivel de plantas procesadoras y en las reempacadoras, porque en los hogares, el valor establecido es de $15 \mathrm{ppm}$ al considerar el hecho de que existe una pérdida de yodo en la sal entre el sitio de producción y el hogar del 20 \% y otro 20 \% durante el proceso de cocción. Es así como en el estudio de 1994-1998 se reportó que la proporción de sal con una concentración de yodo >20 ppm en las muestras tomadas en los hogares (rango validado para ese momento internacionalmente) fue de $83,7 \%$, pasando a ser de $68,3 \%$ en las muestras analizadas en 1998; mientras que en los estudios centinelas de 1999 y de 2001-2002 se encontró

Tabla 6. Resultados nacionales de yodurias en la población colombiana, ENSIN, 2015

\begin{tabular}{l|c|c|c|c}
\hline \multicolumn{1}{|c|}{ Población } & Número de muestras & $\begin{array}{c}\text { Mediana de yoduria } \\
(\mu \mathrm{g} / \mathrm{L})\end{array}$ & $\begin{array}{c}\text { Deficiencia de yodo } \\
(\%)\end{array}$ & Exceso de yodo (\%) \\
\hline Edad: $1-4$ años & 8097 & 365 & 8,1 & 63,8 \\
\hline Edad: 5-12 años & 16151 & 406,8 & 4,4 & 75,2 \\
\hline Mujeres: 13-49 años & 9828 & 379 & 4,9 & 70,5 \\
\hline
\end{tabular}


Figura 1. Flujograma para la monitorización y seguimiento de políticas públicas en los programas de YUS y erradicación de los DDY.

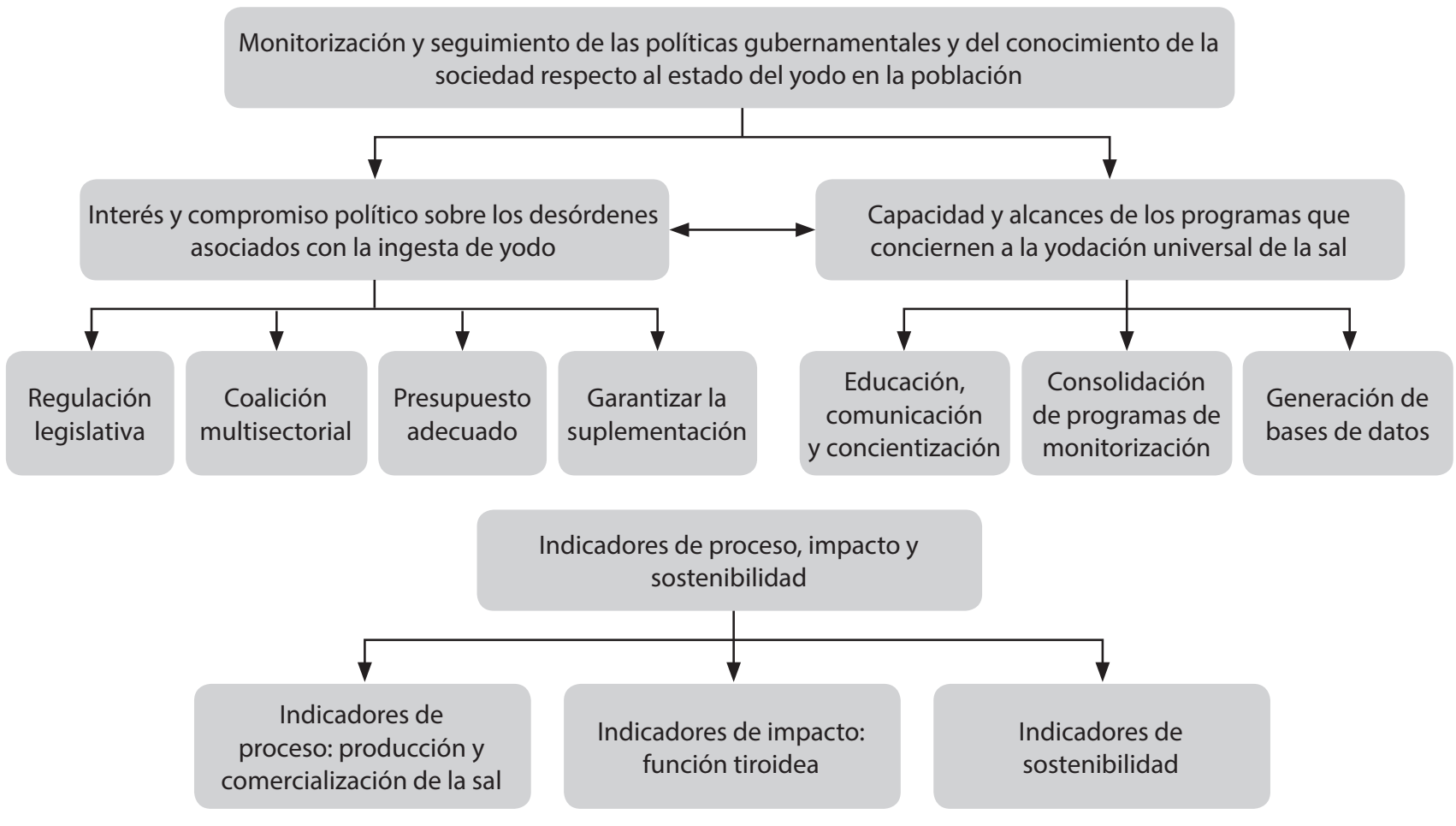

Tabla 7. Países con ingesta excesiva de yodo en la población general, según el reporte de la base de datos global sobre deficiencia de yodo (OMS, 2004)

\begin{tabular}{l|c|c|c|c|c}
\multicolumn{1}{|c|}{ País } & $\begin{array}{c}\text { Total de la población } \\
\text { (año 2002) }\end{array}$ & $\begin{array}{c}\text { Yoduria (mediana, } \\
\boldsymbol{\mu g} / \text { L) }\end{array}$ & $\begin{array}{c}\text { Año de la } \\
\text { encuesta }\end{array}$ & $\begin{array}{c}\text { Población (edad en } \\
\text { años) }\end{array}$ & $\begin{array}{c}\text { Ingesta poblacional } \\
\text { de yodo }\end{array}$ \\
\hline Brasil & 176257000 & 360 & 2000 & Escolares (6-12) & Excesiva \\
\hline Chile & 15613000 & 984 & 2001 & Escolares (6-18) & Excesiva \\
\hline Ecuador & 12810000 & 420 & 1999 & Escolares & Excesiva \\
\hline Liberia & 3239000 & 321 & 1999 & Escolares (6-11) & Excesiva \\
\hline Uganda & 25004000 & 310 & 1999 & Escolares (6-12) & Excesiva \\
\hline
\end{tabular}

que el 73,6 \% y el 90,4\%, respectivamente, tuvieron contenidos >25 ppm; y el 81,8 \% y 98,6\%, respectivamente, tenían contenidos mayores de $15 \mathrm{ppm}$. En todo caso, se redujo la proporción de muestras con niveles no admisibles para el hogar y, por tanto, daba cumplimiento al indicador (al contar con una proporción de hogares $>90 \%$ que consumían sal eficazmente yodada). De acuerdo con lo anterior, debe recordarse que para proporcionar $150 \mu \mathrm{g} /$ día de yodo por medio de la sal, la concentración en el punto de la producción debe estar dentro del intervalo de 20-40 mg de yodo/kg de sal (20-40 ppm, equivalente a 34-66 mg de yodato de potasio/kg de sal).

Cuando la totalidad de la sal que se usa en los alimentos procesados es yodada, se recomienda escoger el límite inferior de concentración, es decir, $20 \mathrm{mg}(51,52)$. En estas circunstancias, la mediana de la concentración de yodo variaría entre 100 y 200 $\mu \mathrm{g} / \mathrm{L}$. Por tanto, si el indicador en la evaluación de la reducción de los DDY establece que $<50 \%$ de las medianas de yodurias estén por debajo de $100 \mu \mathrm{g} / \mathrm{L}$, que $<20 \%$ de las medianas de yo- 
Tabla 8. Países con ingesta excesiva de yodo en la población general y en embarazadas, según la Red Global de Yodo, 2017

\begin{tabular}{|c|c|c|c|c|c|c|}
\hline País & $\begin{array}{l}\text { Total de la } \\
\text { población } \\
\text { (año 2015) }\end{array}$ & $\begin{array}{c}\text { Yoduria } \\
\text { (mediana, } \\
\mu \mathrm{g} / \mathrm{L} \text { ) }\end{array}$ & $\begin{array}{l}\text { Año de la } \\
\text { encuesta }\end{array}$ & $\begin{array}{c}\text { Población (edad en } \\
\text { años) }\end{array}$ & $\begin{array}{l}\text { Ingesta pobla- } \\
\text { cional de yodo }\end{array}$ & $\begin{array}{c}\text { Mediana de yoduria } \\
\text { en embarazadas, año } \\
\text { de encuesta }\end{array}$ \\
\hline Armenia & 3017712 & 313 & 2005 & Escolares (8-10) & Excesiva & NR \\
\hline Benín & 10879829 & 318 & 2011 & Escolares (6-12) & Excesiva & NR \\
\hline Colombia & 48228704 & 415 & 2002 & Escolares & Excesiva & $\begin{array}{c}354 \text { mg/L, año 2013- } \\
2014 \text { (ingesta más que } \\
\text { adecuada) }\end{array}$ \\
\hline Costa Rica & 4807850 & 314 & $\begin{array}{l}2008- \\
2009\end{array}$ & Escolares & Excesiva & NR \\
\hline Yibuti & 887861 & 335 & 2015 & Escolares & Excesiva & $\begin{array}{c}265 \mu \mathrm{g} / \mathrm{L} \text {, año } 2015 \\
\text { (ingesta más que } \\
\text { adecuada) }\end{array}$ \\
\hline Georgia & 3999812 & 321 & 2005 & Escolares (6-12) & Excesiva & NR \\
\hline Honduras & 8075060 & 356 & 2005 & Escolares & Excesiva & NR \\
\hline Catar & 2235355 & 341 & 2014 & Escolares (6-12) & Excesiva & NR \\
\hline Islas Salomón & 583591 & 328 & 2007 & Escolares (6-12) & Excesiva & NR \\
\hline Somalia & 10787104 & 417 & 2009 & Escolares & Excesiva & NR \\
\hline Uganda & 39032383 & 464 & 2005 & Escolares (6-12) & Excesiva & NR \\
\hline
\end{tabular}

NR: no reportada.

durias estén por debajo de $50 \mu \mathrm{g} / \mathrm{L}$ (y que la mediana de las yodurias, por tanto, sean $>100 \mu \mathrm{g} / \mathrm{L}$ ), Colombia cumpliría a cabalidad con el indicador (de acuerdo con el estudio de 1994-1998 y de los 3 estudios centinelas realizados), pero se encontraría muy por encima de estos valores, tal y como se demuestra en los estudios más recientes (Tablas 5, 6 y 8). De esta forma, si se mantuviesen los niveles de ingesta de sal, y si la concentración de yodo en la sal se estableciera en un rango de 20-40 ppm (20$40 \mathrm{mg}$ de yodo/kg de sal), esto sería suficiente para garantizar el suministro de yodo en la población.

Adicionalmente, si se disminuyera la ingesta de sal a $<5 \mathrm{~g} /$ persona/día, sería congruente con una concentración de yodo cercana a 40 ppm; no obstante, dado que a los alimentos producidos comercialmente se les agrega sal antes de su venta, y como cada vez se reemplazan más los alimentos preparados en el hogar, donde el uso de la sal yodada es discrecional (puesto que se agrega en la mesa y al cocinar), es importante entonces que los alimentos comerciales contengan las cantidades proporcionales de yodo necesarias para satisfacer las necesidades poblacionales (Tabla 9). Por esta razón, las diferentes organizaciones internacionales recomendaron una concentración de yodo en la sal de 20-40 ppm en el lugar de producción (suponiendo una ingesta promedio de sal per cápita de $10 \mathrm{~g} /$ persona/día) y se recomendó que la ingesta promedio poblacional de sal (procedente de todas las fuentes alimentarias) fuera $<5 \mathrm{~g} /$ persona/día (con el fin de reducir la ingesta de sodio a menos de $2 \mathrm{~g}$ /persona/día).

Por su parte, la sal de los alimentos se considera también como un factor de riesgo asociado con la presencia de enfermedades crónicas diferentes a la enfermedad tiroidea como cáncer gástrico, osteoporosis, litiasis renal, hipertensión arterial (HTA) y, por ende, con el riesgo de muerte y de comorbilidades asociadas como cardiopatía isquémica, ataque vascular cerebral, nefropatía hipertensiva, entre otras. Se calcula que si la sal proveniente de los alimentos se reduce a los niveles recomendados previamente, la prevalencia de HTA se disminuiría en un $30 \%$ (reducciones leves en las cifras de presión arterial disminuyen las tasas de mortalidad por ataque vascular cerebral y por cardiopatía isquémica; por lo que, si se reduce globalmente el consumo de sal proveniente de los alimentos en al menos un $15 \%$ a lo largo de 10 años, se podría evitar entre 8 a 9 millones de muertes) $(31,53-56)$. 
Tabla 9. Concentraciones sugeridas para la fortificación de la sal con yodo

\begin{tabular}{c|c}
\hline $\begin{array}{c}\text { Consumo estimado de sal, } \\
\text { (g/día) }\end{array}$ & $\begin{array}{c}\text { Cantidad promedio de } \\
\text { yodo a agregar en ppm } \\
\text { (mg/kg de sal) }\end{array}$ \\
\hline 3 & 65 \\
\hline 4 & 49 \\
\hline 5 & 39 \\
\hline 6 & 33 \\
\hline 7 & 28 \\
\hline 8 & 24 \\
\hline 9 & 22 \\
\hline 10 & 20 \\
\hline 11 & 18 \\
\hline 12 & 16 \\
\hline 13 & 15 \\
\hline 14 & 14 \\
\hline
\end{tabular}

Para referenciar el consumo de sodio en Colombia, es necesario remitirse a los resultados suministrados por el estudio multicéntrico INTERSALT, llevado a cabo por la OMS (19851987), en el que se encontró que la ingesta diaria promedio de sodio fue de 5,3 g (equivalente a 13,7 g de sal/día en hombres), y de 3,9 g de sodio/día (equivalente a 10,1 g de sal/día en mujeres) (57). Con dichos resultados, y de acuerdo con otros estudios más recientes en Colombia, es lógico plantear que el exceso de yoduria documentado es causado por el alto consumo de sal proveniente de los alimentos; motivo por el cual deberían implementarse todas las estrategias posibles para la reducción de dicho consumo en la población.

En Colombia, solo hasta mediados de la década de 1960 se pudo disminuir la prevalencia de bocio a $<5 \%$, hasta entonces, las prevalencias se habían establecido en $>30 \%$, con posteriores variaciones desde la implementación de los programas de YUS; estos estudios se limitaban a medir la prevalencia de bocio, pero no medían los niveles de yoduria. Los estudios más recientes han determinado la prevalencia de bocio, niveles de yoduria, títulos de anti-TPO, anti-Tg, anticuerpos antirreceptores de tirotropina (TRAb), entre otros parámetros, y han demostrado que existe una ingesta excesiva de yodo (únicamente en un estudio se encontró una ingesta más que adecuada y en otro no se discriminaron puntualmente los valores de yoduria $\geq 100 \mu \mathrm{g} / \mathrm{L}$ ), lo cual puede explicar la alta tasa de bocio y la alta prevalencia en la positividad de anti-TPO y anti-Tg (enfermedad tiroidea autoinmune).
Finalmente, por una parte, podría plantearse que la alta tasa de bocio ha tenido como causa los dos extremos de ingesta de yodo (antes de la implementación de los programas de YUS la causa era la deficiencia de yodo y, desde dicha implementación, podría asumirse que el exceso en la ingesta se ha asociado con el aumento en dicha tasa); no obstante, debe plantearse también la posibilidad de ciertos bociógenos como responsables, al menos en parte, de la alta frecuencia de bocio. Por otra parte, el único estudio realizado en embarazadas hasta el momento no encontró un aumento en la positividad de anti-TPO, pero la prevalencia de bocio fue superior al $25 \%$. Esta baja prevalencia en los niveles de anti-TPO puede ser explicada, al menos en parte, por factores nutricionales (ingesta de selenio, zinc, cobre, manganeso, vitamina D), los cuales pueden modular la secreción de dichos anticuerpos $(58,59)$.

Llama la atención que en aquellos estudios que evalúan la ingesta de yodo de acuerdo con el área geográfica (urbana y rural) demuestran que en el área urbana la ingesta siempre ha sido mayor que en el área rural, y en el área rural se han documentado niveles desde insuficientes hasta excesivos. Estas diferencias pueden explicarse por varios factores, como el empobrecimiento de las zonas rurales, la falta de seguimiento y control de los programas de YUS, ingesta de sal no yodada y baja concentración de yodo en el suelo donde se establecen estas poblaciones. Colombia es una región predominantemente montañosa, donde sus suelos, por sus características, y en muchos casos por las lejanas distancias a las zonas costeras, puede explicar, al menos parcialmente, las diferencias en las yodurias, en especial en áreas rurales. Otros factores asociados pueden ser la presencia de bociógenos de la dieta y la exposición a pesticidas $(60,61)$.

\section{Conclusión}

Los efectos sobre la salud del déficit o del exceso de yodo deben enfocarse a través de pautas equilibradas entre los programas de YUS y de aquellas que conlleven a la reducción del consumo de sal; las dos estrategias que deben plantearse son la disminución de la concentración de yodo en la sal (20-40 ppm) y la reducción del consumo promedio de sal ( $<5 \mathrm{~g} /$ día). Ambas estrategias son compatibles, rentables y de gran beneficio para la población; asimismo, se deben establecer las colaboraciones necesarias y pertinentes para que estos objetivos sean congruentes; además, las autoridades responsables de la ejecución y vigilancia de los programas de YUS deben ajustar el nivel de yodo en la sal. Estas recomendaciones han demostrado que el consumo de sal puede reducirse sin poner en peligro los esfuerzos de fortificación con micronutrientes, particularmente en aquellas áreas donde la ingesta es más que adecuada o excesiva, y donde dicha intervención cumplió fehacientemente con los objetivos planteados de reducción de la tasa de bocio, pero en las que se ha originado un exceso en el riesgo de otras complicaciones. 


\section{Referencias}

1. Bouga M, Lean MEJ, Combet E. Contemporary challenges to iodine status and nutrition: the role of foods, dietary recommendations, fortification and supplementation. Proc Nutr Soc. 2018;77(3):302-13.

2. Velasco I, Bath SC, Rayman MP. Iodine as Essential Nutrient during the First 1000 Days of Life. Nutrients. 2018;10(3). pii: E290.

3. Resolution WHO. WHA58.24. Sustaining the elimination of iodine deficiency disorders. In: Fifty-eighth World Health Assembly, Geneva, 25 May 2005. Geneva: WHO; 2005.

4. Yngve A, Tseng M. Salt: importance in iodine deficiency and sodium excess. Public Health Nutr. 2010;13(5):599-600.

5. Charlton K, Skeaff S. Iodine fortification: why, when, what, how, and who? Curr Opin Clin Nutr Metab Care. 2011;14(6):618-24.

6. Eastman CJ, Zimmermann MB. The Iodine Deficiency Disorders. [Updated 2018 Feb 6]. In: Feingold KR, Anawalt B, Boyce A, et al., editors. Endotext [Internet]. South Dartmouth (MA): MDText.com, Inc.; 2000-. Available from: https://www.ncbi.nlm.nih.gov/books/NBK285556/

7. Prabhu SR, Kannan S, Mahadevan S. Iodine Deficiency: An under Recognized Problem. Indian J Endocrinol Metab. 2017;21(4):640-1.

8. Katagiri R, Yuan X, Kobayashi S, Sasaki S. Effect of excess iodine intake on thyroid diseases in different populations: A systematic review and meta-analyses including observational studies. PLoS One. 2017;12(3):e0173722.

9. Farebrother J, Zimmermann MB, Andersson M. Excess iodine intake: sources, assessment, and effects on thyroid function. Ann N Y Acad Sci. 2019;1446(1):44-65.

10. Wang B, He W, Li Q, Jia X, Yao Q, Song R, et al. U-shaped relationship between iodine status and thyroid autoimmunity risk in adults. Eur J Endocrinol. 2019;181(3):255-266.

11. Woeber KA. Iodine and thyroid disease. Med Clin North Am. 1991;75(1):169-78.

12. Calil-Silveira J, Serrano-Nascimento C, Kopp PA, Nunes MT. Iodide excess regulates its own efflux: a possible involvement of pendrin. Am J Physiol Cell Physiol. 2016;310(7):576-82.

13. Markou K, Georgopoulos N, Kyriazopoulou V, Vagenakis AG. Iodine-Induced hypothyroidism. Thyroid. 2001;11(5):501-10.

14. Eng PH, Cardona GR, Fang SL, Previti M, Alex S, Carrasco N, Chin WW, et al. Escape from the acute Wolff-Chaikoff effect is associated with a decrease in thyroid sodium/iodide symporter messenger ribonucleic acid and protein. Endocrinology. 1999;140(8):3404-10.

15. Koukkou EG, Roupas ND, Markou KB. Effect of excess iodine intake on thyroid on human health. Minerva Med. 2017;108(2):136-46.

16. De la Vieja A, Santisteban P. Role of iodide metabolism in physiology and cancer. Endocr Relat Cancer. 2018;25(4):R225-R245.

17. Yildirim Simsir I, Cetinkalp S, Kabalak T. Review of Factors Contributing to Nodular Goitre and Thyroid Carcinoma. Med Princ Pract. 2020;29(1):1-5.

18. Cao LZ, Peng XD, Xie JP, Yang FH, Wen HL, Li S. The relationship between iodine intake and the risk of thyroid cancer: A meta-analysis. Medicine (Baltimore). 2017;96(20):e6734.

19. Kim HJ, Kim NK, Park HK, Byun DW, Suh K, Yoo MH, et al. Strong association of relatively low and extremely excessive iodine intakes with thyroid cancer in an iodine-replete area. Eur J Nutr. 2017;56(3):965-71.

20. Lv C, Yang Y, Jiang L, Gao L, Rong S, Darko GM, et al. Association between chronic exposure to different water iodine and thyroid cancer: A retrospective study from 1995 to 2014. Sci Total Environ. 2017;609:735-41.

21. Parra H. Simple goiter in Colombia. Am J Publ Health. 1948;38:820-4.

22. Góngora López J. Bocio simple y sal yodada en Colombia. Revista de Higiene (Bogotá) 1951;4:291-338.

23. Góngora A, López J, Mújica CF. Dos años de tratamiento del bocio simple con sal yodada en el departamento de Caldas. Medicina y Cirugía.1952;16:341-77.

24. Rueda R, Pardo F La prevención del bocio endémico en Colombia. Boletín de la oficina sanitaria panamericana. 1966:495-503.

25. Carrillo JC. Evaluación de Desórdenes por Deficiencia de Yodo (DDI) en dos poblaciones de Casanare Colombia, 1984-1986. Bogotá: Nestlé, Servicio de información científica; 1988. Universidad Nacional de Colombia, Facultad de Medicina. ISBN-13: 978-8432223662.

26. Prevalencia de los Desórdenes por Deficiencia de Yodo e Ingestión Promedio de Sal. Colombia, 1994-1998. 1. a edición. Bogotá: Ministerio de Salud, Instituto Nacional de Salud, Instituto Colombiano de Bienestar Familiar, Sociedad Colombiana de Endocrinología, UNICEF-OPS/OMS, Colciencias; 2001.

27. Vargas-Uricoechea H, Sierra-Torres $\mathrm{CH}$, Holguín-Betancourt CM, Cristancho-Torres L. Iodine-deficiency disorders. Permanent surveillance of vulnerable zones is poor. Medicina. (Bogotá).2012;34(2):119-44.

28. Control de los Desórdenes por deficiencia de Yodo en Colombia. Evaluación Externa. Ministerio de Salud/OPS/OMS. 1998:3-8.

29. Definición del problema para el análisis de impacto normativo relacionado con sal para consumo humano [internet]. Ministerio de Salud y Protec- ción Social; 2019 [acceso el 17 de agosto de 2019]. Disponible en: https:// www.minsalud.gov.co/Normativa/PublishingImages/Paginas/analisis de-impacto-normativo/AIN_Sal.pdf

30. Eliminación sostenible de los desórdenes por deficiencia de yodo en Latinoamérica. Iodine, Unicef, OPS [acceso el 17 de agosto de 2019]. Disponible en: http://www.ign.org/cm_data/Eliminacion_Sostenible_-_Espaol_Doc.pdf

31. Vargas-Uricoechea H, Pinzón-Fernández MV, Bastidas-Sánchez BE, JojoaTobar E, Ramírez-Bejarano LE, Murillo-Palacios J. Iodine Status in the Colombian Population and the Impact of Universal Salt Iodization: A DoubleEdged Sword? J Nutr Metab. 2019;2019:6239243.

32. Córdoba CA, Carrillo JC, Marín A. Current Status of Endemic Goiter and Salt Iodization in Colombia. En: Dunn JT, Pretell EA, Daza CH, Viteri F (editores). Towards the eradication of Endemic Goiter, Cretinism and Iodine Deficiency. PAHO/WHO; 1986. p. 280.

33. Control de los desórdenes por deficiencia de Yodo en Colombia. Evaluación Externa. Ministerio de Salud/OPS/OMS. 1998:3-8.

34. Vigilancia de concentraciones de yodo en sal, Colombia. Estudios centinela de yodo y flúor-fase I-2001. Semana Epidemiológica. 2001;49.

35. Gallego ML, Loango N, Londoño AL, Landázuri P. Niveles de excreción urinaria de yodo en escolares del Quindío, 2006-2007. Rev Salud Pública. 2009;11(6):952-60.

36. Cuéllar-Cuéllar AA. Desórdenes por deficiencia de yodo: bocio endémico correlación entre la presencia de bocio y factores de crecimiento [internet] Universidad Nacional de Colombia; 2010 [acceso el 18 de noviembre de 2017]. Disponible en: https://core.ac.uk/download/pdf/11055934.pdf

37. Londoño AL, Gallego ML, Bayona A, Landázuri P. Hypothyroidism prevalence and its relationship to high levels of thyroid peroxidase antibodies and urinary iodine in a population aged 35 and over from Armenia, 20092010. Rev Salud Pública (Bogotá). 2011;13(6):998-1009.

38. Mora Thiriez S. Situación nutricional de yodo, presencia de bocio y función tiroidea en una población de mujeres embarazadas en la ciudad de Bogotá (Colombia) [internet]. Bogotá: Universidad Nacional de Colombia; 2015 [acceso el 17 de noviembre de 2017]. Disponible en: http://www.bdigital. unal.edu.co/46629/1/05599139.2015.pdf

39. Vargas-Uricoechea H, Bastidas-Sánchez B, Perdomo-Cabrera M, Vargas-Sierra H. Estado nutricional del yodo. Implicación en la positividad de anticuerpos antitiroideos y posible autoinmunidad tiroidea en una población escolar declarada "libre de desórdenes por deficiencia de yodo". Medicina (Bogotá). 2015;37(2):122-39.

40. WHO/UNICEF/ICCIDD. Indicators for assessing iodine deficiency disorders and their control through salt iodization [internet]. Geneva: World Health Organization; 1994 [acceso el 12 de agosto de 2017]. Disponible en: http://whqlibdoc.who.int/hq/1994/WHO_NUT_94.6.pdf

41. Endemic goitre [internet]. Geneva: World Health Organization; 1960 [acceso el 5 de agosto de 2017]. Disponible en: http://whqlibdoc.who.int/ monograph/WHO_MONO_44.pdf

42. WHO/UNICEF/ICCIDD. Assessment of iodine deficiency disorders and monitoring their elimination: a guide for programme managers [actualizado el 1 de septiembre de 2008]. 3. ${ }^{\text {a }}$ edición. Génova: World Health Organization; 2007.

43. Dunn JT. Towards the eradication of endemic goiter, cretinism, and iodine deficiency. Proceedings of the V Meeting of the PAHO/WHO Technical Group on Endemic Goiter, Cretinism and Iodine Deficiency. Washington DC: Pan American Health Organization; 1986.

44. Lyn Patrick ND. Iodine: Deficiency and Therapeutic Considerations. Altern Med Rev. 2008;13(2):116-27.

45. Andersson M, de Benoist B, Rogers L. Epidemiology of iodine deficiency: Salt iodisation and iodine status. Best Pract Res Clin Endocrinol Metab. 2010;24(1):1-11.

46. Zimmermann MB, Andersson M. Update on iodine status worldwide. Cur Opin Endocrinol Diabetes Obes. 2012;19(5):382-7.

47. Andersson M, Karumbunathan V, Zimmermann MB. Global iodine status in 2011 and trends over the past decade. J Nutr. 2012;142(4):744-50.

48. Zimmermann MB, Andersson M. Assessment of iodine nutrition in populations: past, present, and future. Nutr Rev. 2012;70(10):553-70.

49. Iodine Global Network. Global Scorecard of Iodine Nutrition in 2017 in the general population and in pregnant women (PW). IGN: Zurich: 2017.

50. Guideline: Sodium intake for adults and children. Geneva: World Health Organization (WHO); 2012.

51. Salt reduction and iodine fortification strategies in public health [internet]. Geneva: World Health Organization; 2014 [acceso el 12 de septiembre de 2019]. http://apps.who.int/iris/bitstream/10665/101509/1/9789241506694 eng.pdf?ua=1

52. MacGregor GA, He FJ, Pombo-Rodrigues S. Food and the responsibility deal: how the salt reduction strategy was derailed. BMJ. 2015;350:h1936.

53. Bhattacharya U, Chandra AK. Socioeconomic status of the population - a prime determinant in evaluating iodine nutritional status even in a post 
salt iodization scenario. J Pediatr Endocrinol Metab. 2019:32(2):143-9.

54. Santos JAR, Christoforou A, Trieu K, McKenzie BL, Downs S, Billot L, et al. Iodine fortification of foods and condiments, other than salt, for preventing iodine deficiency disorders. Cochrane Database Syst Rev. 2019;2:CD010734

55. Cappuccio FP, Beer M, Strazzullo P; European Salt Action Network. Population dietary salt reduction and the risk of cardiovascular disease. A scientific statement from the European Salt Action Network. Nutr Metab Cardiovasc Dis. 2018;29(2):107-14.

56. He FJ, MacGregor GA. Role of salt intake in prevention of cardiovascular disease: controversies and challenges. Nat Rev Cardiol. 2018;15(6):371-7.

57. Stamler J, Rose G, Stamler R, Elliott P, Dyer A, Marmot M. INTERSALT study findings. Public health and medical care implications. Hypertension. 1989;14(5):570-7.
58. Sun J, Teng D, Li C, Peng S, Mao J, Wang W, et al. Association between iodine intake and thyroid autoantibodies: a cross-sectional study of 7073 early pregnant women in an iodine-adequate region. J Endocrinol Invest. 2020;43(1):43-51.

59. Vargas-Uricoechea H, Bonelo-Perdomo A, Sierra-Torres CH. Iodine and the Thyroid. En: Imam S, Ahmad S (editores) Thyroid disorders: Basic science and clinical practice. Springer; 2016. p. 27-48.

60. Gaitan E. Goitrogens. Baillieres Clin Endocrinol Metab. 1988;2(3):683702.

61. Requena M, López-Villén A, Hernández AF, Parrón T, Navarro Á, Alarcón R. Environmental exposure to pesticides and risk of thyroid diseases. Toxicol Lett. 2019;315:55-63. 


\section{SANOFI}

\section{AYUDE A SUS PACIENTES A LOGRAR LA META DE HbA1c}

$74 \%$ de los pacientes que venían descontrolados con ADOs, alcanzaron la meta de $\mathrm{HbAlc}$ con una sola inyección al día de SOLIQUA ${ }^{\circledR 1}$.

SOLIQUA ${ }^{\circledR}$ reduce en promedio $-2,9 \%$ * de $\mathrm{HbAlc}$ en el sub-grupo de pacientes con $\mathrm{HbAlc}>9 \%$ al inicio del tratamiento?

*Promedio de reducción en pacientes tratados previamente con ADOs con HbAlc > 9\%; el promedio de reducción de HbAlc del total de pacientes descontrolados tratados previamente con ADOs fue -1,6\%.

Conozca más sobre SOLIQUA ${ }^{\boxplus}$ descargando

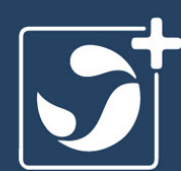

SANOFIMED+

Google play
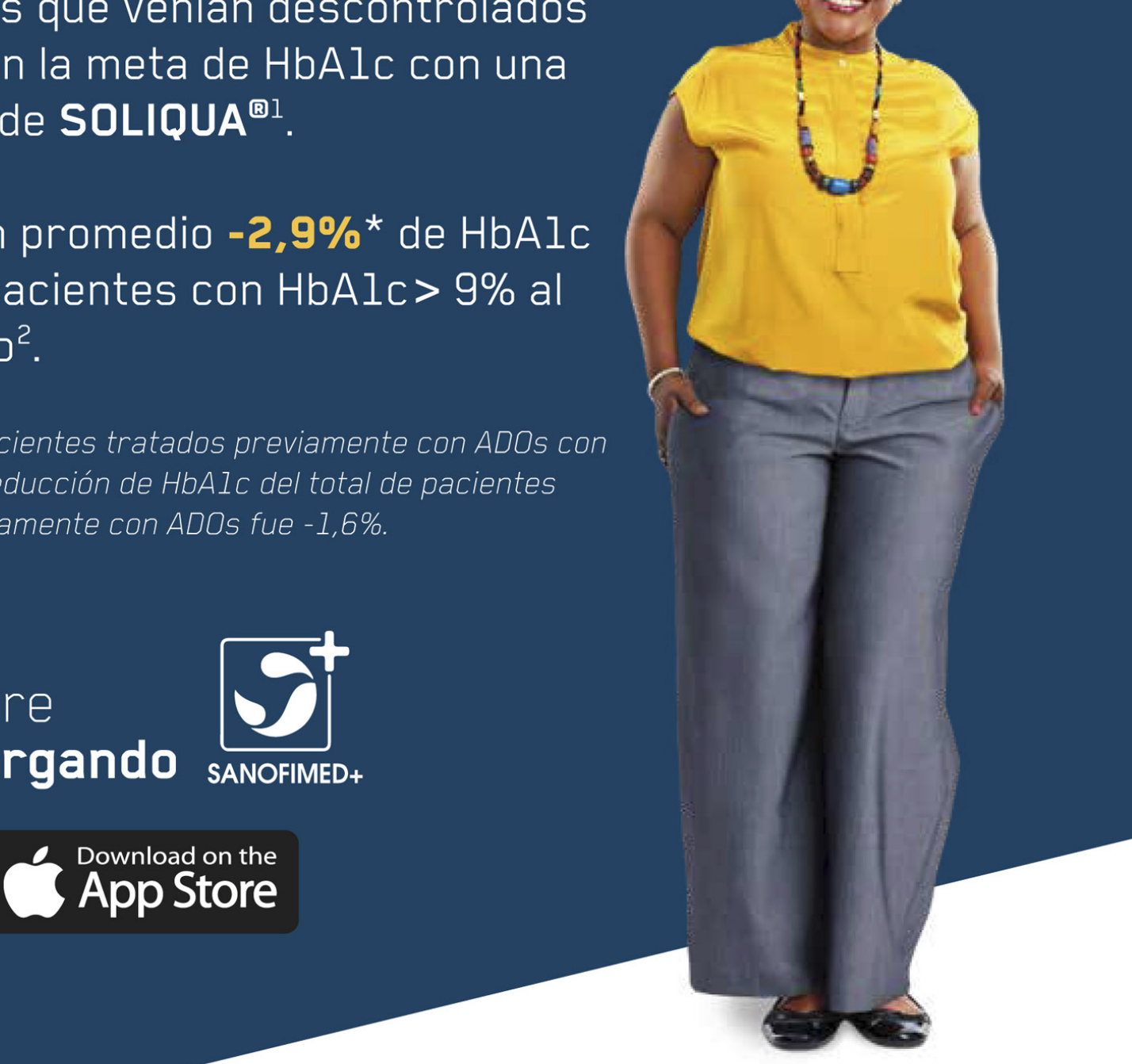

$$
\underset{\text { insulina glargina (100 U/mL) \& lixisenatida }}{\mathbf{S}}
$$

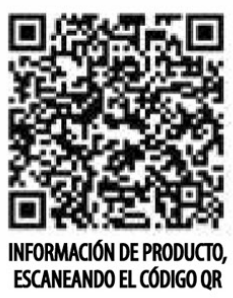

